\title{
Cathepsin B-like and cell death in the unicellular human pathogen Leishmania
}

\author{
AK El-Fadili ${ }^{1}$, H Zangger ${ }^{1}$, C Desponds ${ }^{1}$, IJ Gonzalez ${ }^{1}$, H Zalila ${ }^{1}$, C Schaff ${ }^{1}$, A Ives ${ }^{1}$, S Masina ${ }^{1}$, JC Mottram² and N Fasel ${ }^{\star, 1}$
}

In several studies reporting cell death (CD) in lower eukaryotes and in the human protozoan parasite Leishmania, proteolytic activity was revealed using pan-caspase substrates or inhibitors such as carbobenzoxy-valyl-alanyl-aspartyl-[0-methyl]fluoromethylketone (Z-VAD-FMK). However, most of the lower eukaryotes do not encode caspase(s) but MCA, which differs from caspase(s) in its substrate specificity and cannot be accountable for the recognition of Z-VAD-FMK. In the present study, we were interested in identifying which enzyme was capturing the Z-VAD substrate. We show that heat shock (HS) induces Leishmania $C D$ and leads to the intracellular binding of Z-VAD-FMK. We excluded binding and inhibition of Z-VAD-FMK to Leishmania major metacaspase (LmjMCA), and identified cysteine proteinase C (LmjCPC), a cathepsin B-like (CPC) enzyme, as the Z-VAD-FMK binding enzyme. We confirmed the specific interaction of Z-VAD-FMK with CPC by showing that Z-VAD binding is absent in a Leishmania mexicana strain in which the $c p c$ gene was deleted. We also show that parasites exposed to various stress conditions release CPC into a soluble fraction. Finally, we confirmed the role of CPC in Leishmania CD by showing that, when exposed to the oxidizing agent hydrogen peroxide $\left(\mathrm{H}_{2} \mathrm{O}_{2}\right)$, cpc knockout parasites survived better than wild-type parasites (WT). In conclusion, this study identified CPC as the substrate of Z-VAD-FMK in Leishmania and as a potential additional executioner protease in the CD cascade of Leishmania and possibly in other lower eukaryotes.

Cell Death and Disease (2010) 1, e71; doi:10.1038/cddis.2010.51; published online 2 September 2010

Subject Category: Immunity

In unicellular organisms, several studies reported phenotypic markers of cell death (CD), when cells were exposed to environmental stress such as temperature shock, oxidizing agents, microbial peptides or drugs. ${ }^{1,2}$ Although these reports provided some indication of the existence of a CD pathway in unicellular organisms with similarities to programmed cell death (PCD), experimental evidence of genetically encoded program(s) is still lacking.

Leishmania is an interesting unicellular model organism to study the CD machinery. In the insect vector, Leishmania differentiates from proliferating non-infectious procyclic to non-dividing infectious stationary metacyclic promastigotes. When the parasites are transmitted to its mammalian host, they are phagocytosed by macrophages and differentiate into amastigotes that survive in a phagolysosome. Thus, in both stages, Leishmania parasites must control their growth, maintain their virulence possibly by selecting the fittest parasites and resist the oxidative attack of the innate immune system of the host. ${ }^{3-5}$ Dead or dying promastigotes were shown to enable the intracellular survival of the viable parasites, ${ }^{6,7}$ to influence parasite virulence and modulate the host immune response.

In higher eukaryotes, caspases have a role in the initiation and the execution of the different CD pathways. Plants and lower eukaryotes (e.g., yeast and protozoan parasites such as Leishmania) do not encode caspase(s) but metacaspases (MCA), which could be precursors of caspases. ${ }^{8}$ These cysteine proteases harbor the predicted secondary structure with the catalytic dyad histidine/cysteine ${ }^{9,10}$, and were found to be auto-processed and to recognize mainly substrates with arginine residues in the $\mathrm{P} 1$ position. ${ }^{11-13}$ Thus, MCA have substrate specificities that differ from metazoan caspase usual substrates and are likely not to be accountable for recognition of caspase substrates or inhibitors as reported in several studies. ${ }^{14-17}$

The presence of other proteases acting in the Leishmania CD pathway could explain the recognition of substrates such as carbobenzoxy-valyl-alanyl-aspartyl-[O-methyl]-fluoromethylketone (Z-VAD-FMK), a cell permeate pan-caspase inhibitor. This caspase inhibitor binding was used as a phenotypic marker of CD in yeast cells or protozoan parasites undergoing $C D^{13,18-20}$ and was also shown to block $C D$ in lower eukaryotes such as Dictyostelium. ${ }^{21}$ This lead to a confusing situation as these organisms do not code for caspase.

In this study, we report that Z-VAD-FMK does not inhibit LmjMCA activity but binds to cathepsin B-like (CPC) enzyme, providing an explanation for the detection of caspase

\footnotetext{
${ }^{1}$ Department of Biochemistry, Faculty of Biology and Medicine, University of Lausanne, 155 Chemin des Boveresses, 1066 Epalinges, Switzerland and ${ }^{2}$ Wellcome Centre for Molecular Parasitology and Division of Infection and Immunity, Glasgow Biomedical Research Centre, University of Glasgow, G12 8TA Glasgow, UK ${ }^{*}$ Corresponding author: N Fasel, Department of Biochemistry, University of Lausanne, 155 Chemin des Boveresses, Epalinges 1066, Switzerland. Tel: + 4121 6925732; Fax: + 4121 6925705; E-mail: Nicolas.Fasel@unil.ch

Keywords: cysteine peptidase; cathepsin B; biotin-VAD-FMK; Leishmania; trypanosomes; unicellular organisms

Abbreviations: $\mathrm{CD}$, cell death; $\mathrm{HS}$, heat shock; NO, nitric oxide; $\mathrm{H}_{2} \mathrm{O}_{2}$, hydrogen peroxide; WT, wild-type parasites; $\mathrm{CPC}$, cathepsin B-like; cpc KO, cathepsin B-likedeficient parasites; $c p c \mathrm{KO} /+$, cathepsin B-like-deficient parasites complemented with cpc episomal copy; FITC-VAD-FMK, fluorescein isothiocyanate-valyl-alanylaspartyl-[O-methyl]-fluoromethylketone; Z-VAD-FMK, carbobenzoxy-valyl-alanyl-aspartyl-[O-methyl]-fluoromethylketone; MCA, metacaspase; MOMP, mitochondrial outer membrane permeabilization

Received 19.5.10; revised 20.7.10; accepted 21.7.10; Edited by V De Laurenzi
} 
substrates binding in organisms lacking caspase genes. Furthermore, we provide evidence that CPC is present in a soluble form when CD is induced by various inducers. Based on these data, we propose that CPC has a role in the CD machinery of Leishmania parasites and likely in other lower eukaryotes.

\section{Results}

Heat shock (HS) treatment of Leishmania parasites leads to intracellular binding and accumulation of the caspase inhibitor fluorescein isothiocyanate-valyl-alanyl-aspartyl[O-methyl]-fluoromethylketone (FITC-VAD-FMK). Several studies have reported that dying unicellular organisms bind fluorescent-conjugated caspase inhibitors such as Z-VAD-FMK. To investigate this binding, Leishmania parasites were exposed to a HS treatment at $55^{\circ} \mathrm{C}$ for $10 \mathrm{~min}$ followed by incubation overtime with FITC-VAD-FMK (from 30 min to $2 \mathrm{~h}$ ). FITC-VAD-FMK accumulation was monitored by fluorometry (Figure 1a). No significant binding was observed in untreated parasites (white columns), whereas HS-treated parasites exhibited a rapid entry and accumulation of FITC-VAD-FMK. In addition, Z-VAD-FMK intracellular accumulation is time dependent, reaching a maximum after $30 \mathrm{~min}$ and then decreases overtime. These results were further supported by fluorescence microscopy where FITC-VAD-FMK binding was detected only when parasites were exposed to HS (Figure 1b). To quantify the percentage of a

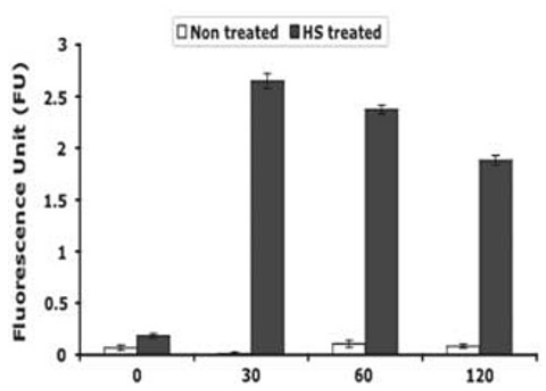

FITC-VAD-FMK Incubation Time (minutes) b

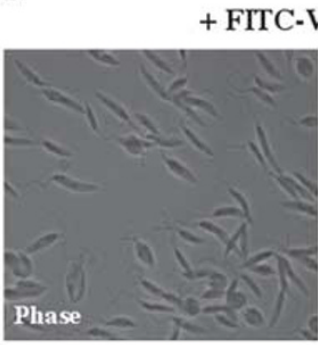

$-\mathrm{HS}$

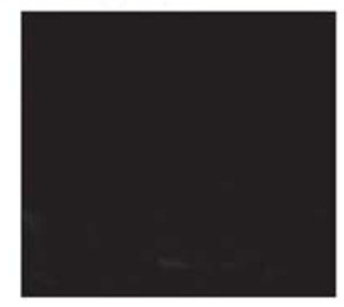

$+\mathrm{HS}$

+ FITC-VAD-FMK (30')

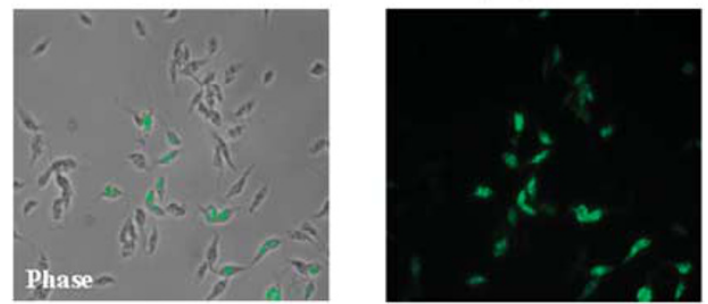

c

-HS
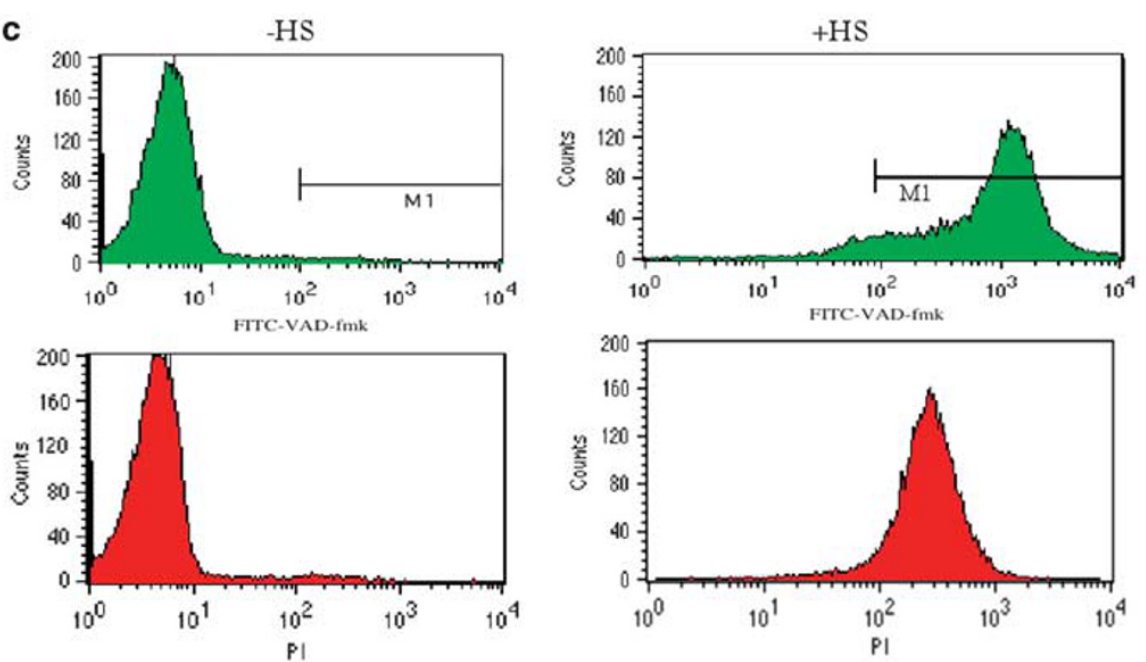

Figure 1 Evaluation of FITC-VAD-FMK binding in Leishmania promastigotes. (a) FITC-VAD-FMK accumulation in L. major wild-type cells was measured after their exposure (HS treated) or not (non-treated) to HS by fluorometry. Fluorescence of intracellular FITC-VAD-FMK was monitored overtime every 30 min for 2 hours. Average of triplicate measurements is shown. (b) FITC-VAD-FMK accumulation was visualized by fluorescence microscopy. After HS exposure (+ HS) or not (-HS), parasites are incubated for 30 min with the FITC-VAD-FMK ( + FITC-VAD $\left(30^{\prime}\right)$ and analyzed by microscopy. (c) Quantification of FITC-VAD-FMK-positive parasites upon HS treatment by flow cytometry. Leishmania WT exposed to HS (+ HS; right panel) compared with untreated parasites ( $-\mathrm{HS}$; left panel) were stained with FITC-VAD-FMK and propidium iodide $(\mathrm{PI})$ 


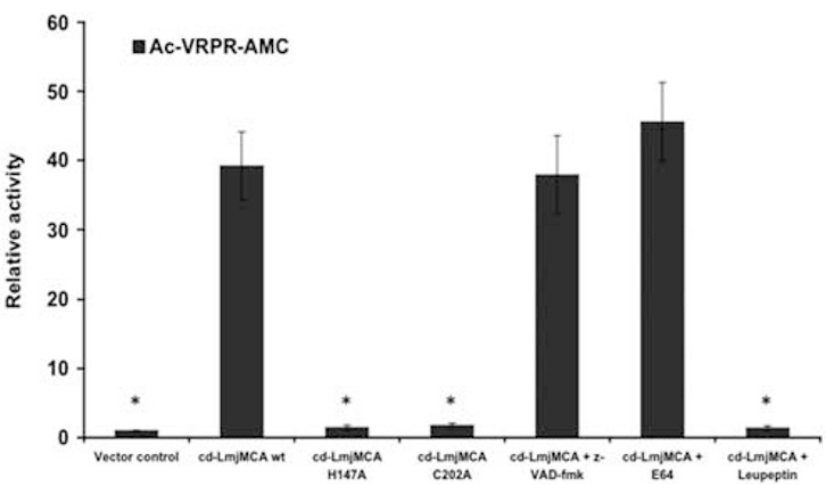

Figure 2 Effect of Z-VAD-FMK, E64 and leupeptin on cd-LmjMCA enzymatic activity. Protein extracts from $\triangle y c a 1$ yeast cells transfected with the $\mathrm{pESC}$-His vector alone (vector control) and expressing the catalytic domain of LmjMCA (cd-LmjMCA) wild type (wt), and its respective H147A and C202A catalytic site mutants were evaluated for their specific activity with the Ac-VRPR-AMC substrate in the absence or the presence of $100 \mu \mathrm{M}$ of Z-VAD-FMK, E64 and leupeptin. The AMC release was measured every $15 \mathrm{~min}$ for $2 \mathrm{~h}$ to determine the activity as the slope of the resulting linear regression. Relative activity was calculated as the fold increase relative to the activity of the vector control (with and without protease inhibitors). Means and S.D. are indicated. ${ }^{*} P<0.05$

parasites binding the FITC-VAD-FMK, we performed a FACS analysis. Over $92 \%$ of the parasites were binding the substrate upon $\mathrm{HS}$ treatment (Figure 1c). Similar results were obtained when hydrogen peroxide $\left(\mathrm{H}_{2} \mathrm{O}_{2}\right)$ or nitric oxide (NO) were used as CD inducers, albeit binding of Z-VAD-FMK was delayed and maximal only after $120 \mathrm{~min}$ (data not shown).

Leishmania MCA peptidase activity is not inhibited by Z-VAD-FMK. Having shown that stressed parasites bind fluorescent-conjugated caspase inhibitors, we wondered whether this inhibitor had any effect on the MCA activity. We tested this possible inhibition of LmjMCA activity by Z-VAD-FMK towards the optimized tetrapeptide substrate VRPR $^{22}$ in a functional heterologous complementation of a Saccharomyces cerevisiae MCA null mutant (4yca1) using the $L$. major MCA catalytic domain (cd-LmjMCAwt). The specificity of LmjMCA for the fluorogenic substrate Ac-Val-Arg-Pro-Arg-7-amino-4-methylcoumarin (Ac-VRPR-AMC) peptide was measured in total protein extracts of $\Delta y c a 1$ cells expressing cd-LmjMCA (Figure 2). As expected, significant activity for cd-LmjMCA with the Ac-VRPR-AMC substrate was obtained when compared with the vector control but activity was completely abrogated when the catalytic dyad was mutated at amino acid histidine 147 or cysteine 202. To be sure that Ac-VRPR-AMC is a sensitive enough substrate, we also compared the levels of activity of cd-LmjMCA with this substrate and other peptidyl substrates previously evaluated. ${ }^{12}$ cd-LmjMCA was found to be twice more active with Ac-VRPR-AMC than with Z-GGR-AMC and almost 10 times more active than with Boc-GRR-AMC (data not shown). These results further confirm that LmjMCA has mainly an arginine-directed cysteine peptidase as reported previously, ${ }^{12}$ closely related to the described plant AtMC9 MCA, and that Ac-VRPR-AMC could be used in our assay to exclude Z-VAD-FMK as an inhibitor of LmjMCA activity.
Total protein extracts of $\Delta y c a 1$ yeast cells expressing cd-LmjMCA were tested for their enzymatic activity with Ac-VRPR-AMC substrate in the presence of high concentrations of the broad caspase inhibitor Z-VAD-FMK, the cysteine protease inhibitor E64, and leupeptin (Figure 2). Z-VAD-FMK and E64 had no significant inhibition of cd-LmjMCA activity when tested with Ac-VRPR-AMC (Figure 2). Interestingly, leupeptin, a serine protease inhibitor, which also inhibits some calpains and cathepsins, completely abrogated cd-LmjMCA activity. These results demonstrate that the VRPR-AMC is a substrate for LmjMCA as for the Arabidopsis thaliana MCA AtMC $9,{ }^{22}$ that cleavage of the substrate is dependent on the catalytic activity of LmjMCA and that this activity is not inhibited by Z-VAD-FMK or E64, known inhibitors of caspase and cathepsin $L$, respectively. The recognition of substrates such as Z-VAD in dying cells might be explained by the binding to other proteases acting in the Leishmania CD pathway.

Biotin-VAD-FMK binds to specific polypeptides in HS-treated Leishmania parasites but does not bind to LmjMCA. To biochemically characterize Z-VAD-FMKbinding polypeptide(s), we replaced FITC-VAD-FMK with a biotin-VAD-FMK (b-VAD-FMK; Figure 3). Untreated parasites incubated in the absence or in the presence of $b-V A D$ FMK were used as controls to detect any binding unrelated to the action of stress inducers or to detect non-specific binding of the avidin-peroxidase (Figure 3a, lanes 1 and 2). When parasites were exposed to HS and subsequently incubated with b-VAD-FMK, several polypeptides of apparent molecular masses of 34 and $47 \mathrm{kDa}$ could be detected by the avidin-peroxidase conjugate (Figure $3 a$ ). No detection of these polypeptides was noted when biotin-VAD-FMK was omitted from the reaction. Considering that these polypeptides migrated with molecular masses in the range of precursor and processed forms of LmjMCA, the same samples were further analyzed for the expression and the processing of LmjMCA using the previously characterized anti-MCA antibody (Figure $3 \mathrm{~b}$ ). ${ }^{12}$ Following HS treatment, LmjMCA precursor is efficiently cleaved generating two main bands with apparent molecular masses of 29 and $45 \mathrm{kDa}$. The band corresponding to the processed catalytic domain of LmjMCA migrating with a molecular mass of $29 \mathrm{kDa}$ differed to that of b-VAD-FMK-detected bands, which migrated with an apparent molecular mass of $34 \mathrm{kDa}$. Similarly, the biotinbinding $47 \mathrm{kDa}$ band migrated slower than the $45 \mathrm{kDa}$ band detected by the anti-MCA antibody (Figure $3 b$ ). Thus, the bVAD-FMK-binding polypeptides differ from Leishmania MCA polypeptides, confirming the results obtained with the LmjMCA activity inhibition experiment (Figure 2).

CPC is the binding partner of the Z-VAD-FMK substrate in $\mathrm{HS}-, \mathrm{H}_{2} \mathrm{O}_{2}-$ or miltefosine-treated Leishmania parasites. To identify the enzyme responsible for b-VADFMK binding, we performed an affinity pull-down of the 'enzyme-biotin-VAD-FMK' complex using an avidin column followed by protein separation on a 1D gel. The gel was stained by Coomassie blue, and the 34 and the $47 \mathrm{kDa}$ stained bands present in the HS-treated samples were isolated and further analyzed using peptide fingerprinting and 

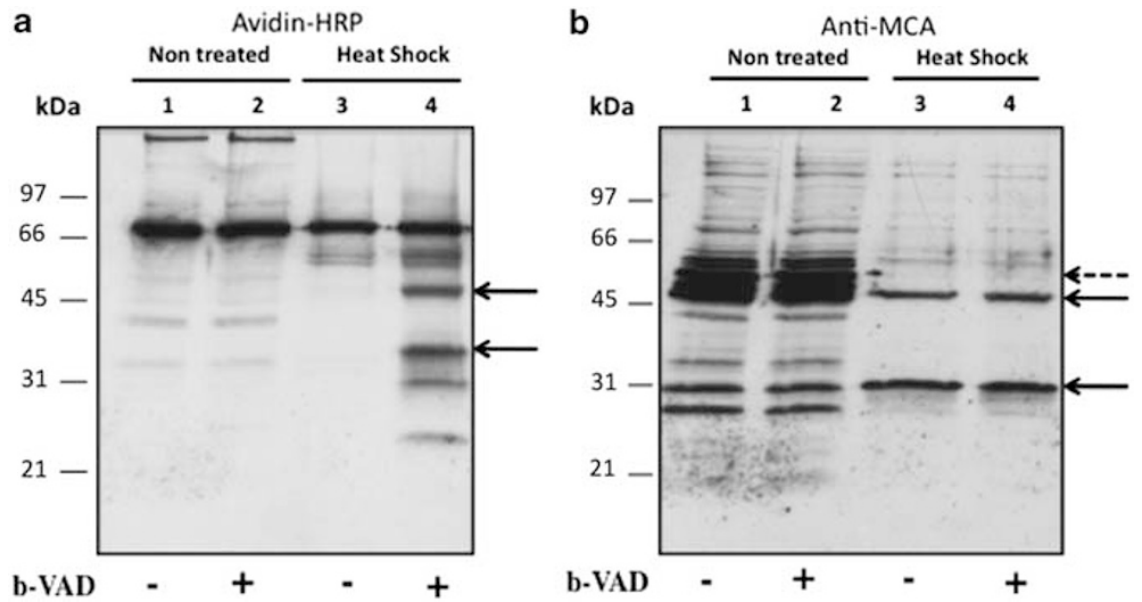

Figure 3 VAD-FMK binds to specific polypeptides that are not related to Leishmania MCA. Western blot analysis of non-treated WT and HS-treated parasites after their incubation $(+b-V A D)$ or not $(-b-V A D)$ with biotin-VAD-FMK (b-VAD). Protein extracts were obtained after parasite lysis using NP-40 buffer and analyzed by immunoblot with avidin-HRP (a) and anti-MCA (b). Black arrows indicate the two bands of 34 and $47 \mathrm{kDa}$, respectively, that were detected to bind the Z-VAD-FMK substrate (a, lane 4). Black dashed arrow indicates full-length LmjMCA (b, lanes 1 and 2) and black arrows indicate lower molecular weight cleavage products of LmjMCA (b, lanes 3 and 4 )

mass spectrometry. Database search for homology at the primary amino acid sequence of the $47 \mathrm{kDa}$ band did not allow us to detect any tryptic peptides corresponding to LmjMCA or to any enzyme which could bind VAD-FMK. In the $34 \mathrm{kDa}$ eluted band, however, we could identify tryptic peptides corresponding to CPC, a CPC enzyme, as a possible b-VAD-FMK-binding polypeptide. CPC has a predicted molecular mass of $37.7 \mathrm{kDa}$ in the precursor form and of $34 \mathrm{kDa}$ in the active processed form. ${ }^{23}$ In the Leishmania genome, CPC is encoded by a single gene LmjF29.0820. To further confirm CPC as the b-VAD-FMKbinding partner, we studied b-VAD-FMK binding in L. mexicana $c p c$ knockout parasites as well as in $c p c$ complemented lines. ${ }^{24}$ After HS treatment and incubation in the presence or in the absence b-VAD-FMK substrate, samples were separated by SDS-PAGE and binding of b-VAD-FMK was revealed by avidin-peroxidase. Binding of b-VAD-FMK was detected only in parasites expressing CPC, for example, in L. mexicana wild type (WT) and in the $c p c$ knockout complemented cell line (cathepsin B-like deficient parasites complemented with $c p c$ episomal copy $(c p c \mathrm{KO} /+))$, respectively (Figure $4 \mathrm{a})$, as revealed by the presence of $34 \mathrm{kDa}$ band. No signal was detected in the $c p c$ knockout parasites (cathepsin B-like-deficient parasites ( $c p c \mathrm{KO}$ ); Figure $4 \mathrm{a}$ ). As expected, the signals corresponded to the expected molecular mass of the CPC processed form. The reason for the presence of a doublet at $34 \mathrm{kDa}$ in HS-treated cells is not known. In HS-treated L. mexicana, no $47 \mathrm{kDa}$ species was detected by the avidin-peroxidase, confirming that this biotin-binding polypeptide is likely to be a non-specific binding of b-VAD-FMK when $L$. major parasites are HS treated.

To confirm that Z-VAD-FMK binding is not restricted to HS-treated parasites, we have analyzed the $c p c$ null mutants using chemical and therapeutic $C D$ inducers. Treated L. mexicana $\mathrm{WT}, c p c \mathrm{KO}$ and $c p c \mathrm{KO} /+$ parasites with $\mathrm{H}_{2} \mathrm{O}_{2}$ were further incubated with b-VAD-FMK and its binding to $\mathrm{CPC}$ was revealed by avidin-peroxidase (Figure $4 \mathrm{~b}$ ).
Similar to $\mathrm{HS}, \mathrm{H}_{2} \mathrm{O}_{2}$ treatment leads to binding of Z-VADFMK to CPC, as the $34 \mathrm{kDa}$ polypeptide is detected only in parasites expressing CPC (Figure 4b, lanes 2 and 6). No signal was visible in $c p c \mathrm{KO}$ cells (Figure $4 \mathrm{~b}$, lane 4).

Second, the binding of Z-VAD-FMK to CPC was also induced by the anti-Leishmania drug miltefosine, a drug known to disrupt the lysosomal membrane (Figure 4c). When parasites were treated overnight with $40 \mu \mathrm{M}$ of miltefosine, followed by b-VAD-FMK incubation, the release of the $34 \mathrm{kDa}$ was visible only in parasites expressing CPC (Figure 4c, lanes 2 and 6). Again, no specific signal was detected in the $c p c \mathrm{KO}$ parasites (Figure 4b, lane 4). These results suggest that CPC is present and released from the lysosome when parasites are exposed to different $C D$ inducers including $\mathrm{HS}, \mathrm{H}_{2} \mathrm{O}_{2}$ or miltefosine and that, at least in our experimental design, the proteolytic activity of CPC, as detected by the binding of b-VAD-FMK substrate, could be involved in the CD pathway of Leishmania parasites.

Finally, to show that the release of CPC from the lysosome occurred following exposure to different inducers, we used a rat antiserum raised against a recombinant $\mathrm{CPC}$, and analyzed CPC expression and release in both treated and untreated cells. Wild-type L. mexicana, $c p c \mathrm{KO}$ and a $c p c$ $\mathrm{KO} /$ + were exposed to $\mathrm{HS}, \mathrm{H}_{2} \mathrm{O}_{2}$ or miltefosine (Figures $4 \mathrm{~d}$, $\mathrm{e}$, and f). Expression and release of CPC was analyzed in a NP-40 soluble fraction. In these experiments, we expected to detect not only the processed CPC enzyme but also its precursor form, if these different treatments provoked the rupture of the fragile lysosomal membrane and consequent release of enzymes into the cytoplasm of the dying parasite. Upon treatment with $\mathrm{HS}, \mathrm{H}_{2} \mathrm{O}_{2}$ or miltefosine, two polypeptides with molecular masses of 34 and $38 \mathrm{kDa}$ were specifically recognized by the anti-CPC antiserum in WT and $c p c \mathrm{KO} /$ + parasites, but not in $c p c \mathrm{KO}$ parasites (Figures $4 \mathrm{~d}$, $e$, and f). These two polypeptides migrated at the expected sizes for CPC precursor and processed forms, respectively. These CPC precursor and processed forms were also detected in untreated parasites albeit at a lower level. 

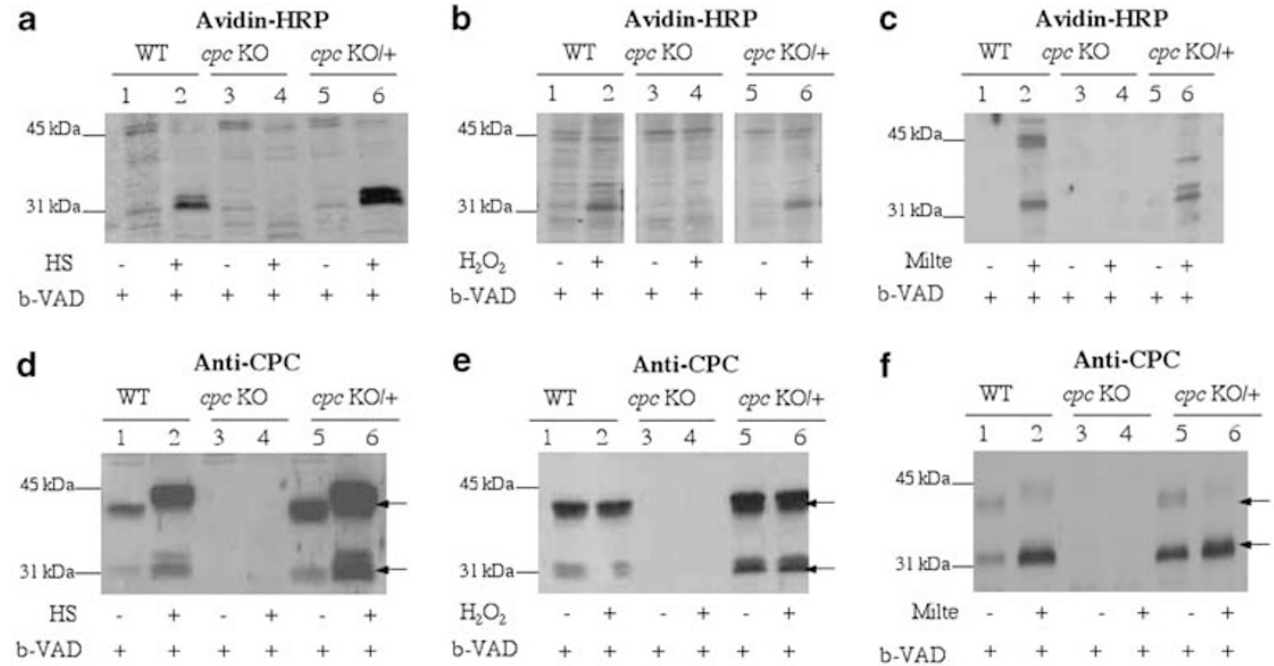

Figure 4 Detection of Z-VAD-FMK binding in CPC-deficient mutants by western blot. (a-c) Parasites' extracts were analyzed for Z-VAD-FMK binding using avidin-HRP. All parasites were exposed $(+)$ or not $(-)$ to $\mathrm{HS}(\mathbf{a}), \mathrm{H}_{2} \mathrm{O}_{2}(\mathbf{b})$ or miltefosine $(\mathbf{c})$, and incubated with biotin-VAD-FMK $(+$ b-VAD). Protein extracts were derived from WT, $c p c \mathrm{KO}$ and $c p c \mathrm{KO}$. (d-f), Total protein extracts from WT, $c p c \mathrm{KO}$ and $c p c \mathrm{KO} /+$ cell lines exposed $(+)$ or not $(-)$ to $\mathrm{HS}(\mathbf{d}), \mathrm{H}_{2} \mathrm{O}_{2}$ (e) or miltefosine (f) and incubated with biotin-VAD-FMK ( + b-VAD) were further analyzed for CPC expression using a CPC anti-serum. Black arrows indicate the two major forms of the CPC protein ( 34 and $38 \mathrm{kDa}$, respectively)

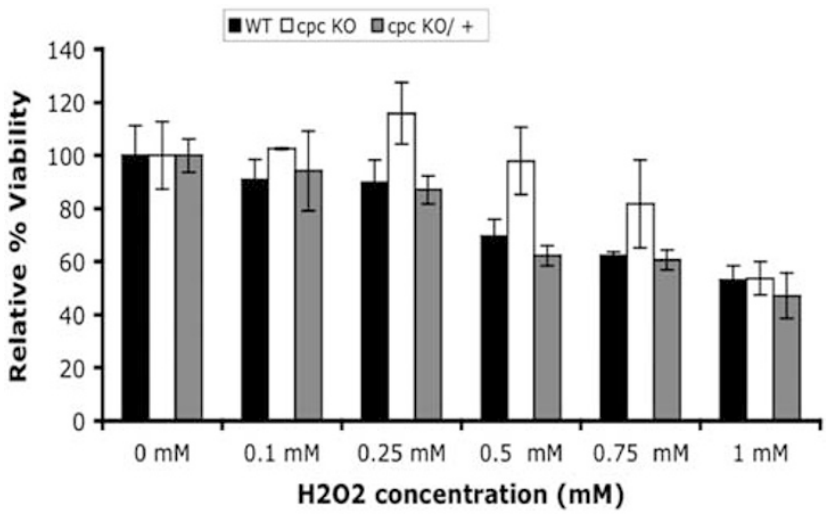

Figure 5 CPC-deficient parasites are more resistant to $\mathrm{H}_{2} \mathrm{O}_{2}$-induced death. WT, $c p c \mathrm{KO}$ and $c p c \mathrm{KO}$ + were measured for viability using the MTS assay. Parasites were treated with increasing concentrations of $\mathrm{H}_{2} \mathrm{O}_{2}$ for 1 hour. Results are a mean of triplicates and expressed as a relative percentage of viability. Experiment was repeated at least three times independently

The reason for such a presence in untreated parasites is likely to be because of the rupture of some lysosomes during the fractionation procedure thereby contaminating the NP-40 soluble fraction.

CPC participates to the Leishmania CD pathway. If CPC is involved in Leishmania $C D$, it is expected that $c p c$ knockout parasites survive better to reactive oxygen species (ROS) or to chemotherapeutics drugs. We, therefore, performed viability tests of $L$. mexicana WT, $c p c$ $\mathrm{KO}$ parasites and $c p c \mathrm{KO} /+$ parasites in the presence of different concentrations of $\mathrm{H}_{2} \mathrm{O}_{2}$. Viability was measured in stationary parasites using the MTS assay. Increasing the concentration of $\mathrm{H}_{2} \mathrm{O}_{2}$ from 0.1 to $1 \mathrm{mM}$ induced the death of over $40 \%$ of the parasites (Figure 5 ) suggesting that, at high concentrations, CD is induced independently of the presence or the absence of CPC. Interestingly, at $0.5 \mathrm{mM}$ of $\mathrm{H}_{2} \mathrm{O}_{2}, c p c$ $\mathrm{KO}$ parasites survive better than parasites expressing CPC and show a higher survival rate (100\%) as compared with WT cells $(70 \%)$ or cpc $\mathrm{KO} /+$ cells $(65 \%$; Figure 5$)$. This result suggests that CPC activation increased the parasites' sensitivity to death signals, as lack of CPC enhanced the parasites' survival to death stimuli.

To provide a more detailed analysis of the CD process, we measured the exposure of phosphatidylserine at the cytoplasmic membrane of L. mexicana WT, CPC KO and $\mathrm{CPC} \mathrm{KO/} \mathrm{+} \mathrm{lines} \mathrm{with} \mathrm{FITC-labeled} \mathrm{Annexin} \mathrm{V.} \mathrm{Parasites} \mathrm{with}$ a loss of integrity in their membrane (necrotic cells) were detected with 7-AAD (Figure 6). When parasites were treated with $1 \mathrm{mM} \mathrm{H}_{2} \mathrm{O}_{2}$ for $1 \mathrm{~h}$ (Figure 6), we noticed that parasites expressing CPC (e.g. WT and $c p c \mathrm{KO} /+$ cells) exhibited a high rate of necrotic (7-AAD +) and late apoptotic (AnV + /7$\mathrm{ADD}+$ ) cells, whereas $c p c$-deficient cells ( $c p c \mathrm{KO})$ show a low percentage of necrotic and late apoptotic cells, as shown by calculating the ratios of percentage of AnV + /7-AAD-, $\mathrm{AnV}+/ 7-\mathrm{AAD}+$ and AnV-/7-AAD + cells to percentage of $A n V-/ 7-A A D-(T a b l e ~ 1)$. These results confirmed that CPCexpressing Leishmania exhibit an enhanced sensitivity to undergo CD. In our assay, this sensitivity is almost abrogated when parasites are lacking CPC. In addition, we noticed that the CD kinetics differ between parasites expressing CPC and the null mutants. When parasites are exposed to $\mathrm{H}_{2} \mathrm{O}_{2}$ (Figure 6, Table 1), both WT and CPC KO/ + show low rate of early apoptotic cells (AnV +/7-AAD-) compared with CPC KO parasites (Figure 6, Table 1). Similar results were obtained when cells were exposed to HS or miltefosine (data not shown). In the light of these results, we can conclude that CPC expression is directly linked to the parasite sensitivity to $C D$ inducers. Thus, parasites lacking CPC are more resistant to the lethal effect of environmental and chemical CD stimuli. Our results also suggest that $C D$ in Leishmania could implicate not only the release of $\operatorname{LmjMCA}^{12}$ 

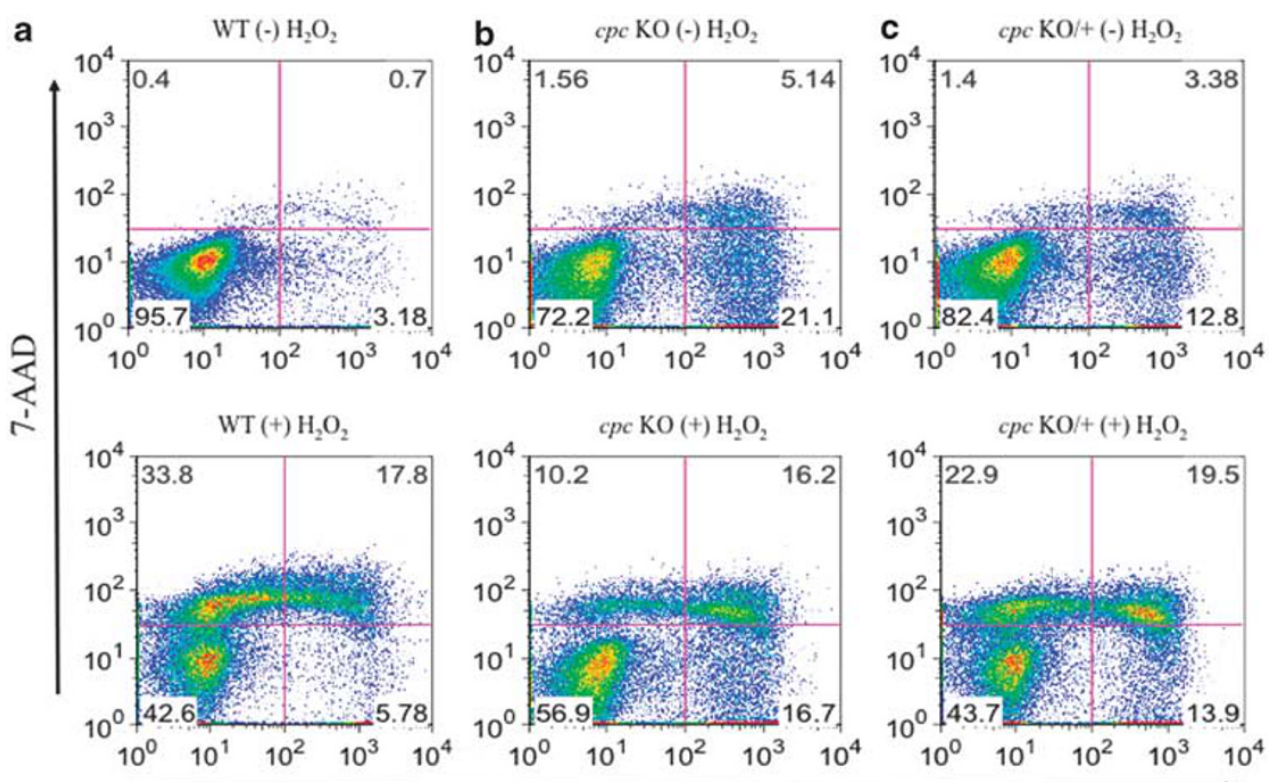

Annexin V

d

WT
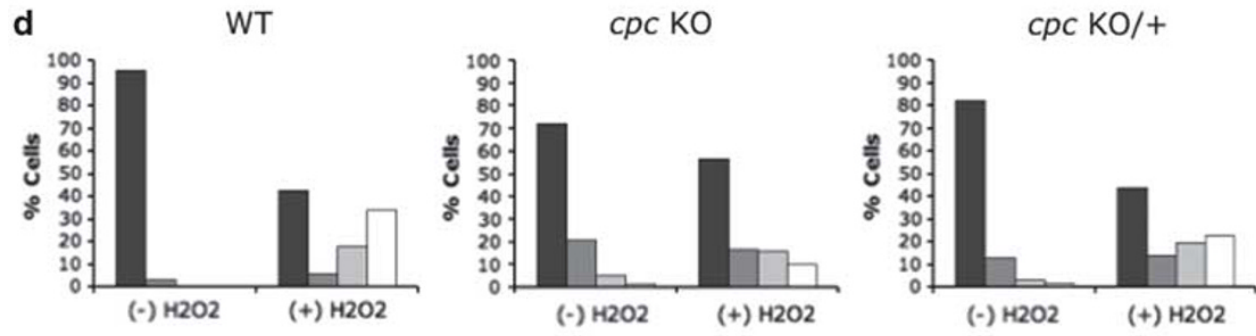

anV (-) 7-AAD (-) $\square$ AnV (+) 7-AAD (-)

$\square \operatorname{AnV}(+)$ 7-AAD (+)

$\square \operatorname{AnV}(-)$ 7-AAD (+)

Figure 6 Phosphatidylserine exposure at the membrane of Leishmania cells treated with $\mathrm{H}_{2} \mathrm{O}_{2}$. WT (column a), $c p c \mathrm{KO}$ (column $\mathbf{b}$ ) and $c p c \mathrm{KO} /+($ column $\mathbf{c}$ ) that contain an episomal copy of $c p c$ were not treated $(-)$ or treated $(+)$ with $1 \mathrm{M} \mathrm{H}_{2} \mathrm{O}_{2}$ for $1 \mathrm{~h}$. Cells were stained with fluorescein isothiocyanate (FITC)-labeled Annexin V and 7-AAD to distinguish between live (AnV-/7AAD-), apoptotic (AnV + /7AAD -, early apoptotic cells; AnV + /7AAD +, late apoptotic cells) and necrotic cells (AnV - /7AAD +). (d) Percentage of AnV-/7AAD -, AnV + /7AAD -, AnV + /7AAD + and AnV-/7AAD + in WT, cpc KO and cpc KO/ + when non-treated (-) or treated (+) with 1 mM $\mathrm{H}_{2} \mathrm{O}_{2}$ for $1 \mathrm{~h}$ after staining with fluorescein isothiocyanate (FITC)-labeled Annexin $\mathrm{V}$ and 7-AAD (a-c)

Table 1 Kinetics of the parasites' death under $\mathrm{H}_{2} \mathrm{O}_{2}$ treatment

\begin{tabular}{|c|c|c|c|}
\hline & $\operatorname{AnV}(+)$ 7-AAD (-) & $\operatorname{AnV}(+)$ 7-AAD (+) & $\operatorname{AnV}(-)$ 7-AAD (+) \\
\hline $\begin{array}{l}\text { Wild-type (-) } \mathrm{H}_{2} \mathrm{O}_{2} \\
\text { Wild-type (+) } \mathrm{H}_{2} \mathrm{O}_{2} \\
\text { CPC KO (-) } \mathrm{H}_{2} \mathrm{O}_{2} \\
\text { CPC KO (+) } \mathrm{H}_{2} \mathrm{O}_{2} \\
\text { CPC KO/+ (-) } \mathrm{H}_{2} \mathrm{O}_{2} \\
\text { CPC KO/+ (+) } \mathrm{H}_{2} \mathrm{O}_{2}\end{array}$ & $\begin{array}{l}0.033 \\
0.14 \\
0.29 \\
0.29 \\
0.16 \\
0.32\end{array}$ & $\begin{array}{l}0.007 \\
0.42 \\
0.07 \\
0.28 \\
0.04 \\
0.45\end{array}$ & $\begin{array}{l}0.004 \\
0.79 \\
0.02 \\
0.18 \\
0.02 \\
0.53\end{array}$ \\
\hline
\end{tabular}

Wild-type parasites (WT), cathepsin B-deficient parasites ( $c p c \mathrm{KO}$ ) and $c p c \mathrm{KO}$ complemented strain ( $c p c \mathrm{KO} /+)$ that contain an episomal copy of $c p c$ were not treated (-) or treated (+) with $1 \mathrm{mM} \mathrm{H}_{2} \mathrm{O}_{2}$ for $1 \mathrm{~h}$. Cells were stained with fluorescein isothiocyanate (FITC)-labeled Annexin $\mathrm{V}$ and 7-AAD to distinguish between live (AnV-/7AAD-), apoptotic (AnV+/7AAD-, early apoptotic cells; AnV+/7AAD+, late apoptotic/necrotic) and necrotic cells (AnV-/7AAD+) as depicted in Figure 6. Values correspond to ratios of percentage of AnV+/7-AAD-, AnV+/7-AAD+ and AnV-/7-AAD+ cells to percentage of AnV-/7-AAD-

but also the release of a soluble form of CPC enzyme. Thus, previous studies showing Z-VAD-FMK binding may be integrated into a model, taking into account a proteolytic cascade, implicating CPC proteolytic activity.

\section{Discussion}

A CD program could control the density of Leishmania population in the insect stage, be involved in the virulence of the inoculum, in the innate immune response when parasites are killed in the host phagolysosome by $\mathrm{NO}$ or $\mathrm{H}_{2} \mathrm{O}_{2}$, in the control of the parasite density in the macrophage and in the chemosensitivity of parasites to specific drugs.

In this study, we decided to solve the confusing issue of detection of the pan-caspase Z-VAD-FMK substrate binding to dying unicellular cells using Leishmania as a model system. After having excluded that $b-V A D-F M K$ binds and inhibits MCA, we demonstrated that b-VAD-FMK is binding to an 
active CPC in stress situations only. These data provide strong evidence that at least part of $C D$ in Leishmania may function via CPC and result in consequent release of cathepsins into the cytoplasm.

Our results are consistent with our previous report showing that, upon induction of $\mathrm{CD}$ by $\mathrm{HS}$ or NO, lysosomal cathepsins $\mathrm{L}$ (i.e., CPA and CPB) were released into the cytoplasm. ${ }^{25}$ However, in the previous study, we did not provide any evidence of the functional role of Leishmania CPA, CPB or CPC. The data presented here suggest that, among CPs, Leishmania CPC is involved in CD. These results do not exclude, however, the participation of CPA and CPB in the proteolytic degradation of the cellular components. As revealed by the inhibition observed with the use of the $\mathrm{CP}$ inhibitor E-64, these CPs could be responsible for the activities toward caspase-like substrates such as the DEVDase activity detected in axenic amastigote-like parasites, but not in the free-living promastigote. ${ }^{25}$ Our results on the function of CPC in CD and the binding of b-VAD-FMK have been obtained in promastigote parasites. In the light of these results, it will be important to evaluate further CPs' activities in the different life stages of the parasite, using different substrates and single null mutants $(\Delta c p a, \Delta c p b$ and $\Delta c p c)$ to provide answers to the respective role of each $\mathrm{CP}$ in the CD pathway.

Our results also explain the detection of $b$-VAD-FMK binding observed in several dying unicellular organisms. Biotin-VAD-FMK binding could not be explained by binding to caspase(s), as caspase genes are absent in these organisms. Thus, binding of Z-VAD-FMK to an active CPC in dying cells could be a general process in the CD pathway in unicellular organisms. In higher eukaryotes, cathepsin B was shown to bind Z-VAD-FMK. Cathepsin B has been shown to bind $b-V A D-F M K$ in other organisms ${ }^{26,27}$ and to be involved in PCD pathways in mammalian cells, arguing in favor of a lysosomal membrane permeabilization implicated in CD. ${ }^{28,29}$ Interestingly, mammalian cathepsins were observed to participate in the apoptotic cascade of hepatocytes, neuronal cells and T lymphocytes. ${ }^{30-32}$ Furthermore, cathepsins were shown to participate directly or indirectly in mitochondrial outer membrane permeabilization (MOMP) with release of cytochrome $c$ and in the activation of caspases 9 and $3 .^{33}$ In other systems, the CP inhibitor E-64 was shown to block PCD in the dinoflagellate Peridinium. ${ }^{34}$ This class of proteases could be, in concert with LmjMCA, one of the main executioner proteases in lower eukaryotes. It is not known yet whether release of lysosomal enzymes is selective as observed in mammalian cells. ${ }^{35,36}$

Our experimental data, using wild-type parasites (WT), $c p c$ $\mathrm{KO}$, and complemented line, tend to demonstrate that when $\mathrm{CPC}$ is present, the percentage of necrotic cells increase. Thus far, we cannot conclude whether we are confronted to a simple necrosis or a programmed necrosis such as necroptosis. ${ }^{37}$ Whether dying unicellular organisms are simply using existing enzymatic activities by default in stress situations (circumstantial death) or whether the CD pathway is genetically encoded needs further studies. The presence of several common mediators in metazoa and in unicellular organisms, as proposed by a bioinformatics comparison, ${ }^{38}$ and the detection of similar phenotypic markers in higher and lower eukaryotes suggest that CD is controlled in unicellular organisms. Although the role of cathepsins and other mediators needs to be studied in other organisms, our data support the hypothesis that PCD or PCD-like was present early in evolution and that PCD in metazoa could have evolved from a common pathway. In this context, one possible common early pathway may have been based on the rupture of the fragile lysosomal membrane by oxidative stress and consequent release of lysosomal enzymes. In this hypothesis, other lysosomal enzymes, and especially the abundant CPs, would be released into the cytoplasm and could participate in the degradation of cellular components. MOMP could be induced by cathepsins, which would lead to the release of cytochrome $c$ and the formation of ROS. The latter would have a permeabilization effect on lysosomal and mitochondrial membranes. ${ }^{31}$ This lysosomal pathway is likely to be used for different stress stimuli, by some antiparasitic drugs such as miltefosine, a drug known to induce apoptotic CD via both the intrinsic mitochondrial pathway and a lysosomal pathway in mammalian cells. ${ }^{39}$

\section{Materials and Methods}

Chemicals. Reagents, unless otherwise stated, were purchased from SigmaAldrich Inc. (St Louis, MO, USA).

Culture of Leishmania promastigotes. L. major MRHO/IR/75/ER promastigotes, $L$. mexicana wild-type cells and $L$. mexicana deletion mutants ( $c p c \mathrm{KO}$ and $c p c \mathrm{KO} /+)^{24}$ were grown until stationary phase at $26^{\circ} \mathrm{C}$ in $\mathrm{M} 199$ medium (Invitrogen AG, Basel, Switzerland) supplemented with 10\% heatinactivated FCS (Seromed GmbH, Wien, Switzerland), $40 \mathrm{mM} \mathrm{N-2-hydroxy-}$ ethylpiperazine-NO-2-ethanesulfonic acid (Hepes, pH 7.0), $100 \mathrm{U} / \mathrm{ml}$ penicillin and $100 \mu \mathrm{g} / \mathrm{ml}$ streptomycin.

FITC-VAD-FMK accumulation assay. $1 \times 10^{8}$ stationary phase parasites were exposed to a $\mathrm{HS}$ at $55^{\circ} \mathrm{C}$ for $10 \mathrm{~min}$ followed by $1 \mathrm{~h}$ incubation at $26^{\circ} \mathrm{C}$ Treated parasites were centrifuged at $1400 \mathrm{~g}$ and washed with $1 \times$ PBS, then further incubated with $10 \mu \mathrm{M}$ FITC-VAD-FMK (Promega Corp., Madison, WI, USA) for different time points. The labeled parasites were centrifuged at $1400 \mathrm{~g}$ and washed with $1 \times$ PBS. Parasites' pellets were resuspended in $1 \times$ PBS and dispensed in 96-well culture plate for fluorescence measurement. Fluorescence was measured using the Muli-Detection Microplate Reader Synergy 2 (Biotek instruments; Witec AG, Littau, Switzerland) at an excitation wavelength of $355 \mathrm{~nm}$ and an emission wavelength of $460 \mathrm{~nm}$. All incubation steps were at $26^{\circ} \mathrm{C}$.

Flow cytometric analysis, confocal and fluorescence microscopy. Stress induced parasites were washed, resuspended in AnnexinV (AnV)-binding buffer (HEPES $10 \mathrm{mM}, \mathrm{NaCl} 150 \mathrm{mM}, \mathrm{CaCl}_{2} 2.5 \mathrm{mM}$ ) at $\mathrm{pH}$ 7.2. Cells were incubated at room temperature (RT) for $15 \mathrm{~min}$ with AnV-FITC (BD Biosciences Pharmingen, Allschwill, Switzerland) at the concentration indicated by the manufacturer. At the moment of acquisition, $0.4 \mathrm{mg} / \mathrm{ml}$ of propidium iodide (BD Biosciences Pharmingen) was added to control and AnV-FITC-labeled samples. Data were collected in a BD FACSCaliburH and analyzed by Cellquest ProH (BD Biosciences, San Jose, CA, USA). A total of 10000 gated events were harvested from each sample. For confocal microscopy, HS-treated parasites were incubated for $30 \mathrm{~min}$ to $5 \mathrm{~h}$ in the presence or absence of $10 \mu \mathrm{M}$ of FITC-VAD-FMK (Promega Corp.) in M199-complemented medium. As a control, the same procedure was followed using untreated parasites. Hence, labeled parasites were washed with $1 \times$ PBS and adhered to poly-L-lysine-treated coverslips. Slides were mounted with Vectashield mounting medium with DAPI (Vector Laboratories, Burlingame, CA, USA). Mounted slides were visualized using a Zeiss LSM 510 UV META Laser Scanning Confocal Microscope.

MCA enzymatic activity. Protein extracts from MCA null mutant ( $\Delta y c a$ 1) yeast cells transfected with the $p E S C$-His vector alone (vector control) or expressing either the catalytic domain of LmjMCA (cd-LmjMCA) wildtype (wt), or its respective 
H147A and C202A catalytic mutants were evaluated for their specific activity toward the fluorogenic peptidyl substrate Ac-VRPR-AMC. Transfected yeast cells were harvested following $24 \mathrm{~h}$ induction with $2 \%$ galactose. Total protein extracts were prepared in a lysis buffer containing $50 \mathrm{mM} \mathrm{KH}_{2} \mathrm{PO}_{4},(\mathrm{pH} 7.5), 500 \mathrm{mM} \mathrm{NaCl}, 1 \mathrm{mM}$ EDTA, $5 \mathrm{mM}$ DTT and $1 \%$ CHAPS. Enzymatic activity was then evaluated with $40 \mu \mathrm{g}$ of total protein diluted in $200 \mu \mathrm{l}$ of activity buffer $(25 \mathrm{mM}$ HEPES, $150 \mathrm{mM}$ $\mathrm{NaCl}, 10 \mathrm{mM} \mathrm{CaCl}, 10 \%$ glycerol, $0.1 \%$ CHAPS and $10 \mathrm{mM} \mathrm{DTT}$ ) and incubated with $100 \mu \mathrm{M}$ of the Ac-VRPR-AMC. ${ }^{12}$ MCA activity toward Ac-VRPR-AMC was tested in the presence of $100 \mu \mathrm{M}$ of Z-VAD-FMK, E64 or leupeptin. In all cases, the release of AMC was measured at each $10 \mathrm{~min}$ for $2 \mathrm{~h}$ by spectrofluorometry using an excitation wavelength of $355 \mathrm{~nm}$ and an emission wavelength of $460 \mathrm{~nm}$. Enzymatic activity was determined as the slope of the resulting linear regression and expressed as the fold increase relative to the activity of the vector control.

Biotin-VAD-FMK-binding assay. $1.5 \times 10^{8}$ stationary phase parasites were treated with three different death inducers such us $\mathrm{H}_{2} \mathrm{O}_{2}(1 \mathrm{mM}$ for $1 \mathrm{~h})$, $\mathrm{HS}\left(10 \mathrm{~min}\right.$ at $55^{\circ} \mathrm{C}$, followed by $1 \mathrm{~h}$ incubation at $\left.26^{\circ} \mathrm{C}\right)$ or miltefosine $(40 \mu \mathrm{M}$ for $24 \mathrm{~h}$ ). Treated parasites were centrifuged at $1400 \times g$ and washed with $1 \times$ PBS, then further incubated with $100 \mu \mathrm{M}$ biotin-VAD-FMK (Promega Corp.) for $20 \mathrm{~min}$ at RT. The parasites were then centrifuged at $1400 \mathrm{~g}$ and washed with $1 \times$ PBS and pellets were resuspended in cold NP-40 lysis buffer $\left(0.14 \mathrm{M} \mathrm{NaCl}, 1.5 \mathrm{mM} \mathrm{MgCl}_{2}\right.$, $10 \mathrm{mM}$ Tris- $\mathrm{HCl}, 0.5 \%$ Nonidet P40, $2 \mathrm{mM}$ EDTA, $40 \mathrm{ng} / \mathrm{ml}$ leupeptin, $10 \mu \mathrm{g} / \mathrm{ml}$ pepstatin, $0.8 \mathrm{mM}$ o-phenanthroline and $160 \mu \mathrm{M}$ E64). Parasite lysates were centrifuged at $13200 \times g$ at $4^{\circ} \mathrm{C}$ for $3 \mathrm{~min}$. Protein concentration was quantified using a BCA protein assay reagent (Pierce Biotechnology, Inc., Rockford, IL, USA) with BSA as a standard. Cleared lysates and their respective pellets were stored at $-70^{\circ} \mathrm{C}$ for further analysis.

\section{Purification of the enzyme-Biotin-VAD-FMK complex and MS analysis. IR75 parasites were grown $\left(3-4 \times 10^{7} / \mathrm{ml}\right)$, exposed to $\mathrm{HS}$ for $10 \mathrm{~min}$ at $55^{\circ} \mathrm{C}$ and then left at RT for $1 \mathrm{~h}$. After washing the parasites in $1 \times$ PBS, they were resuspended in $200 \mu \mathrm{l}$ of $1 \times$ PBS and $100 \mu \mathrm{l}$ of biotinyl-VAD(Ome)-FMK from Alexis for $20 \mathrm{~min}$ at RT, washed once in $1 \times$ PBS, resuspended in $200 \mu \mathrm{l}$ of NP-40 lysis buffer in the presence of protease inhibitors, left for $1 \mathrm{~min}$ on ice and centrifuged at $13200 \times g$ for $3 \mathrm{~min}$. The supernatant was diluted $4 \times$ in $1 \times$ PBS, and $60 \mu \mathrm{l}$ of streptavidin-agarose beads prewashed in $1 \times$ PBS was added, incubated for $30 \mathrm{~min}$ at RT on a wheel. The beads were recovered after centrifugation, washed three times with one volume of $1 \times$ PBS. One volume of $1 \times$ PBS was added to the volume of the beads. The beads were incubated at $94^{\circ} \mathrm{C}$ for $5 \mathrm{~min}$ in $1 \times$ Laemmli's gel sample buffer and the total was loaded on a $10 \%$ SDS-polyacrylamide gel. The gel was stained by Coomassie blue and stained bands were cut and analyzed by mass spectrometry after trypsin digestion. ${ }^{40}$}

Cell lysis and immunobloting. Stationary promastigotes were lysed in a solution containing $0.14 \mathrm{M} \mathrm{NaCl}, 1.5 \mathrm{mM} \mathrm{MgCl}_{2}, 10 \mathrm{mM}$ Tris- $\mathrm{HCl}, 0.5 \%$ Nonidet P40, $2 \mathrm{mM}$ EDTA, $40 \mathrm{ng} / \mathrm{ml}$ leupeptin, $10 \mu \mathrm{g} / \mathrm{ml}$ pepstatin, $0.8 \mathrm{mM}$ o-phenanthroline and $160 \mu \mathrm{M}$ E64. Protein concentration was quantified using a BCA protein assay reagent (Pierce Biotechnology, Inc.) with BSA as a standard. Twelve percent SDS-PAGEs were used to separate $10-20 \mu \mathrm{g}$ of total protein from parasite lysates. Proteins were transferred to a nitrocellulose membrane by electroblotting and probed with either avidin HRP antibody (BD Biosciences), rat polyclonal antibodies raised against the $L$. major $C P C$ or rabbit polyclonal antibodies produced against a synthetic 76-mer amino acid peptide (ER53) from the caspase-like domain of the Trypanosoma brucei TbMCA2/3 MCA. ${ }^{12}$ Filters were exposed to horseradish peroxidase-conjugated secondary antibodies (Promega Corp.) and developed by enhanced chemiluminescent staining using ECL western blotting system (Amersham Biosciences, Piscataway, NJ, USA). Low-range molecular weight standards were used as reference proteins (Bio-Rad Laboratories, Hercules, CA, USA).

Test for apoptotic markers. Annexin $\mathrm{V}$ staining was performed as described previously ${ }^{12}$ with some modifications. Briefly, $1 \times 10^{7}$ stationary promastigotes either exposed or not for $1 \mathrm{~h}$ to $1 \mathrm{mM}$ of $\mathrm{H}_{2} \mathrm{O}_{2}$ were washed and resuspended in Annexin V binding buffer (10 mM HEPES (pH 7.4), $140 \mathrm{mM} \mathrm{NaCl}$, $2.5 \mathrm{mM} \mathrm{CaCl}_{2}$ and $1.2 \mathrm{M}$ sorbitol). Parasites were incubated in the dark for $15 \mathrm{~min}$ at RT with $5 \mu \mathrm{l}$ Annexin V-FITC (Invitrogen AG). Before FACS analysis, $2.5 \mu \mathrm{l}$ of $7-A A D$ was added to the samples in a final volume of $250 \mu \mathrm{l}$. In all, 50000 events were accumulated with a BD FACScan apparatus and data were analyzed using the
CellQuest (Becton-Dickinson Biosciences, San Jose, CA, USA) and Flowjo (Tree Star Inc., Ashland, OR, USA) softwares.

MTS assay. The toxicity of $\mathrm{H}_{2} \mathrm{O}_{2}$ for the parasites was determined $1 \mathrm{~h}$ after exposure to the oxidative stress agent using the MTS tetrazolium (Promega Corp.). In all, $2 \times 10^{6}$ parasites were dispensed in 96-well culture plate and exposed or not to $1 \mathrm{mM} \mathrm{H}_{2} \mathrm{O}_{2}$ for $1 \mathrm{~h}$. $\mathrm{H}_{2} \mathrm{O}_{2}$ action was stopped by addition of 50 units of catalase to the samples. Samples in the well plates were treated using $50 \mu$ lof MTS tetrazolium and incubated for $2-3 \mathrm{~h}\left(26^{\circ} \mathrm{C}\right)$ in a $5 \% \mathrm{CO}_{2}$ incubator. Once coloring developed, the absorbance of $200 \mu \mathrm{l}$ of each sample was analyzed at $490 \mathrm{~nm}$ using the Muli-Detection Microplate Reader Synergy2 (Biotek instruments, Witec AG).

Statistical analysis. All the experiments were performed at least three times independently, and means and S.D. were calculated. The Student's $t$-test was used in statistical analysis.

\section{Conflict of interest}

The authors declare no conflict on interest.

Acknowledgements. We thank J Mauel for careful reading of the paper and C Ronet for help in the analysis of the FACS data. This work was supported by grants from the FNRS No. 3100A0-116665/1, the Novartis Foundation, the Dr Rub foundation and the Swiss Secretariat for Education and Research in the framework of the COST action BM0802 to NF, and the Medical Research Council (Grant no. G0700127) to JCM.

1. Shaha C. Apoptosis in Leishmania species \& its relevance to disease pathogenesis. Indian $J$ Med Res 2006; 123: 233-244.

2. Deponte M. Programmed cell death in protists. Biochim Biophys Acta 2008; 1783: 1396-1405.

3. Moore KJ, Matlashewski G. Intracellular infection by Leishmania donovani inhibits macrophage apoptosis. J Immunol 1994; 152: 2930-2937.

4. Moore KJ, Turco SJ, Matlashewski G. Leishmania donovani infection enhances macrophage viability in the absence of exogenous growth factor. J Leukoc Biol 1994; 55: 91-98.

5. Ouaissi A, Ouaissi M. Molecular basis of Trypanosoma cruzi and Leishmania interaction with their host(s): exploitation of immune and defense mechanisms by the parasite leading to persistence and chronicity, features reminiscent of immune system evasion strategies in cancer diseases. Arch Immunol Ther Exp (Warsz) 2005; 53: 102-114.

6. van Zandbergen G, Bollinger A, Wenzel A, Kamhawi S, Voll R, Klinger M et al. Leishmania disease development depends on the presence of apoptotic promastigotes in the virulent inoculum. Proc Natl Acad Sci USA 2006; 103: 13837-13842.

7. Wanderley JL, Pinto da Silva LH, Deolindo P, Soong L, Borges VM, Prates DB et al. Cooperation between apoptotic and viable metacyclics enhances the pathogenesis of leishmaniasis. PLoS One 2009; 4: e5733.

8. Carmona-Gutierrez D, Eisenberg T, Buttner S, Meisinger C, Kroemer G, Madeo F. Apoptosis in yeast: triggers, pathways, subroutines. Cell Death Differ 2010; 17: 763-773.

9. Uren AG, O'Rourke K, Aravind LA, Pisabarro MT, Seshagiri S, Koonin EV et al. Identification of paracaspases and metacaspases: two ancient families of caspase-like proteins, one of which plays a key role in MALT lymphoma. Mol Cell 2000; 6: 961-967.

10. Aravind L, Dixit VM, Koonin EV. Apoptotic molecular machinery: vastly increased complexity in vertebrates revealed by genome comparisons. Science 2001; 291: 1279-1284.

11. Vercammen D, van de Cotte B, De Jaeger G, Eeckhout D, Casteels P, Vandepoele $K$ et al. Type II metacaspases Atmc4 and Atmc9 of Arabidopsis thaliana cleave substrates after arginine and lysine. J Biol Chem 2004; 279: 45329-45336.

12. Gonzalez IJ, Desponds C, Schaff C, Mottram JC, Fasel N. Leishmania major metacaspase can replace yeast metacaspase in programmed cell death and has arginine-specific cysteine peptidase activity. Int J Parasitol 2007; 37: 161-172.

13. Watanabe N, Lam E. Two Arabidopsis metacaspases AtMCP1b and AtMCP2b are arginine/lysine-specific cysteine proteases and activate apoptosis-like cell death in yeast. J Biol Chem 2005; 280: 14691-14699.

14. Das M, Mukherjee SB, Shaha C. Hydrogen peroxide induces apoptosis-like death in Leishmania donovani promastigotes. J Cell Sci 2001; 114: 2461-2469.

15. Sen N, Das BB, Ganguly A, Mukherjee T, Bandyopadhyay S, Majumder HK. Camptothecin-induced imbalance in intracellular cation homeostasis regulates programmed cell death in unicellular hemoflagellate Leishmania donovani. J Biol Chem 2004; 279: 52366-523675.

16. Arnoult D, Akarid K, Grodet A, Petit PX, Estaquier J, Ameisen JC. On the evolution of programmed cell death: apoptosis of the unicellular eukaryote Leishmania major involves cysteine proteinase activation and mitochondrion permeabilization. Cell Death Differ 2002; 9: 65-81. 
17. Paris C, Loiseau PM, Bories C, Breard J. Miltefosine induces apoptosis-like death in Leishmania donovani promastigotes. Antimicrob Agents Chemother 2004; 48: 852-859.

18. Madeo F, Herker E, Maldener C, Wissing S, Lachelt S, Herlan M et al. A caspase-related protease regulates apoptosis in yeast. Mol Cell 2002; 9: 911-917.

19. Bozhkov PV, Filonova LH, Suarez MF, Helmersson A, Smertenko AP, Zhivotovsky B et al. VEIDase is a principal caspase-like activity involved in plant programmed cell death and essential for embryonic pattern formation. Cell Death Differ 2004; 11: 175-182.

20. Al-Olayan EM, Williams GT, Hurd H. Apoptosis in the malaria protozoan, Plasmodium berghei: a possible mechanism for limiting intensity of infection in the mosquito. Int J Parasitol 2002; 32: 1133-1143.

21. Olie RA, Durrieu F, Cornillon S, Loughran G, Gross J, Earnshaw WC et al. Apparent caspase independence of programmed cell death in Dictyostelium. Curr Biol 1998; 8: 955-958.

22. Vercammen D, Belenghi B, van de Cotte B, Beunens T, Gavigan JA, De Rycke R et al. Serpin1 of Arabidopsis thaliana is a suicide inhibitor for metacaspase 9. J Mol Biol 2006 ; 364: 625-636.

23. Lecaille F, Kaleta J, Bromme D. Human and parasitic papain-like cysteine proteases: their role in physiology and pathology and recent developments in inhibitor design. Chem Rev 2002; 102: 4459-4488

24. Bart G, Frame MJ, Carter R, Coombs GH, Mottram JC. Cathepsin B-like cysteine proteinasedeficient mutants of Leishmania mexicana. Mol Biochem Parasitol 1997; 88: 53-61.

25. Zangger $\mathrm{H}$, Mottram JC, Fasel N. Cell death in Leishmania induced by stress and differentiation: programmed cell death or necrosis? Cell Death Differ 2002; 9: 1126-1139.

26. Rozman-Pungercar J, Kopitar-Jerala N, Bogyo M, Turk D, Vasiljeva O, Stefe I et al. Inhibition of papain-like cysteine proteases and legumain by caspase-specific inhibitors: when reaction mechanism is more important than specificity. Cell Death Differ 2003; 10 : 881-888.

27. Schotte $P$, Declercq W, Van Huffel S, Vandenabeele $P$, Beyaert R. Non-specific effects of methyl ketone peptide inhibitors of caspases. FEBS Lett 1999; 442: 117-121.

28. Boya P, Kroemer G. Lysosomal membrane permeabilization in cell death. Oncogene 2008; 27: $6434-6451$.

29. Berndtsson M, Beaujouin M, Rickardson L, Havelka AM, Larsson R, Westman J et al. Induction of the lysosomal apoptosis pathway by inhibitors of the ubiquitin-proteasome system. Int J Cancer 2009; 124: 1463-1469.

30. Conus S, Simon HU. Cathepsins: key modulators of cell death and inflammatory responses. Biochem Pharmacol 2008; 76: 1374-1382.
31. Chwieralski CE, Welte T, Buhling F. Cathepsin-regulated apoptosis. Apoptosis 2006; 11: 143-149.

32. Michallet MC, Saltel F, Flacher M, Revillard JP, Genestier L. Cathepsin-dependen apoptosis triggered by supraoptimal activation of T lymphocytes: a possible mechanism of high dose tolerance. J Immunol 2004; 172: 5405-5414.

33. Guicciardi ME, Leist M, Gores GJ. Lysosomes in cell death. Oncogene 2004; 23 . 2881-2890.

34. Vardi A, Berman-Frank I, Rozenberg T, Hadas O, Kaplan A, Levine A. Programmed cell death of the dinoflagellate Peridinium gatunense is mediated by $\mathrm{CO}(2)$ limitation and oxidative stress. Curr Biol 1999; 9: 1061-1064.

35. Cirman T, Oresic K, Mazovec GD, Turk V, Reed JC, Myers RM et al. Selective disruption of Iysosomes in HeLa cells triggers apoptosis mediated by cleavage of Bid by multiple papainlike lysosomal cathepsins. J Biol Chem 2004; 279: 3578-3587.

36. Conus S, Perozzo R, Reinheckel T, Peters C, Scapozza L, Yousefi S et al. Caspase-8 is activated by cathepsin $D$ initiating neutrophil apoptosis during the resolution of inflammation. J Exp Med 2008; 205: 685-698.

37. Christofferson DE, Yuan J. Necroptosis as an alternative form of programmed cell death Curr Opin Cell Biol 2010; 22: 263-268.

38. Nedelcu AM. Comparative genomics of phylogenetically diverse unicellular eukaryotes provide new insights into the genetic basis for the evolution of the programmed cell death machinery. J Mol Evol 2009; 68: 256-268.

39. Paris C, Bertoglio J, Breard J. Lysosomal and mitochondrial pathways in miltefosineinduced apoptosis in U937 cells. Apoptosis 2007; 12: 1257-1267.

40. Walker J, Acestor N, Gongora R, Quadroni M, Segura I, Fasel N et al. Comparative protein profiling identifies elongation factor-1beta and tryparedoxin peroxidase as factors associated with metastasis in Leishmania guyanensis. Mol Biochem Parasitol 2006; 145: 254-264

Cell Death and Disease is an open-access journal published by Nature Publishing Group. This work is licensed under the Creative Commons Attribution-Noncommercial-No Derivative Works 3.0 Unported License. To view a copy of this license, visit http://creativecommons.org/licenses/by-nc-nd/3.0/ 